\title{
Stability Analysis of Train Movement with Uncertain Factors
}

\author{
JingJing Ye, ${ }^{1}$ KePing Li, ${ }^{2}$ and XueDong Jiang ${ }^{1}$ \\ ${ }^{1}$ School of Electrical Engineering, Beijing Jiaotong University, Beijing 100044, China \\ ${ }^{2}$ State Key Laboratory of Rail Traffic Control and Safety, Beijing Jiaotong University, Beijing 100044, China \\ Correspondence should be addressed to KePing Li; kpli@bjtu.edu.cn
}

Received 27 May 2015; Revised 22 July 2015; Accepted 11 August 2015

Academic Editor: Yuanchang Xie

Copyright (C) 2015 JingJing Ye et al. This is an open access article distributed under the Creative Commons Attribution License, which permits unrestricted use, distribution, and reproduction in any medium, provided the original work is properly cited.

\begin{abstract}
We propose a new traffic model which is based on the traditional OV (optimal velocity) car-following model. Here, some realistic factors are regarded as uncertain quantity, such as the headway distance. Our aim is to analyze and discuss the stability of carfollowing model under the constraint of uncertain factors. Then, according to the principle of expected value in fuzzy theory, an improved OV traffic model is constructed. Simulation results show that our proposed model can avoid collisions effectively under uncertain environment, and its stability can also be improved. Moreover, we discuss its stability as some parameters change, such as the relaxation time.
\end{abstract}

\section{Introduction}

In the past 60 years, the theory of traffic flow has been gradually developed and improved. Now, it becomes the foundation which is used to explain complex traffic phenomena, solve some difficult traffic problems, and increase the efficiency of traffic system. A number of theoretical calculations, numerical simulations, and empirical observations are reported. Even so, the theory of traffic flow still faces many great challenges of some realistic traffic problems.

In the theory of traffic flow, car-following model is an important microscopic simulation model which has been widely extended and applied. Since the car-following model uses realistic driver behavior and detailed vehicle characteristics, it can be used to simulate various traffic phenomena which are observed in realistic traffic [1-3], such as soliton wave, shock wave, and kink wave. A famous car-following model is optimal velocity model which was proposed by Bando et al. in 1995 [4]. Afterwards, many improved and extended car-following models were reported which were based on Bando's model. For example, Helbing and Tilch found that collision would occur if accelerating rate is too large; then they derived general force model (GF) [5]. Jiang et al. studied start process of static motorcade by GF and proposed full velocity difference model [6]. Li et al. studied stabilization strategies of relaxation time of driver based on general nonlinear car-following model [7].

Usually, in railway traffic, there are two kinds of block modes for controlling the train movement: the fixed block and moving block modes. With the fixed block mode, the train movement is controlled by signal lights. However, with the moving block mode, one train runs following its leading train with the help of wireless communication. Moving block mode which is based on communication is the most advanced signal control system in the world. At present, it is extensively used in the urban railway traffic and high-speed railway traffic. With the moving block mode, train runs at higher speed and smaller time gap. Figure 1 displays the principle of train movement with moving block mode.

The key step with moving block mode is about the safety of train movement. In Figure 1, the safety of train movement is determined by headway distance between leading train 2 and following train 1 :

$$
\begin{aligned}
& L_{A}=L_{B}+L_{Z}, \\
& L_{B}=\tau v+\frac{v^{2}}{\left(2 b_{b r}\right)} .
\end{aligned}
$$

Here, $L_{A}$ is the safe headway distance, $\tau$ is relaxation time, $v$ is the velocity of train movement, and $b_{b r}$ is the maximum 


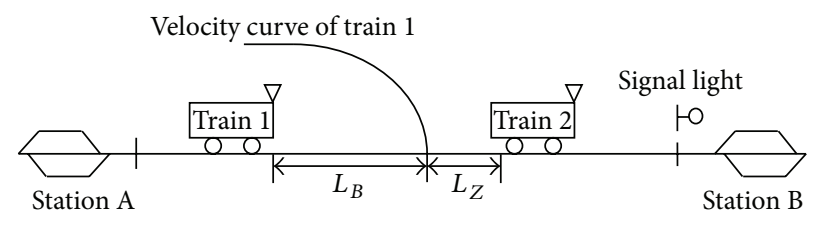

Figure 1: The Moving Block Principle.

deceleration. $L_{Z}$ is a margin which cannot be less than the length of whole train.

In realistic traffic, vehicle's movement is under the constraint of many uncertain factors, such as weather, driver's judgment, and passenger demand. Recently, such a problem attracts more and more attention. Zheng et al. studied the urban pickup and delivery problem in which the velocities of vehicles were regarded as fuzzy and uncertain [8]. In 2010, Chen and Zhou proposed a new model called the reliable mean-excess traffic equilibrium model, in which it reflected driver's risk preferences under uncertain environment [9]. Zheng and Van Zuylen proposed a probabilistic delay distribution model with stochastic arrivals and departures [10]. In railway traffic, the passenger demand and waiting time usually were considered as uncertain factors, which were treated as fuzzy variables [11]. Wei et al. proposed locationscheduling model based on genetic algorithm, where transportation risk was considered as time-dependent fuzzy random variables [12]; Yang et al. studied credibility-based rescheduling model in a double-track railway network, where low-probability incident was taken as uncertain factor [13].

In present work, the headway distance of vehicle is regarded as fuzzy variable. Based on traditional OV carfollowing model, we propose a new OV car-following model. Since we have accounted for some uncertain factors, our proposed model is more realistic than traditional model. This paper is organized as follows. In Section 2, traditional OV car-following model is introduced, our proposed model is proposed in Section 3, and simulation results are shown in Section 4. Finally, a conclusion is drawn in Section 5.

\section{Optimal Velocity Car-Following Model}

In principle, car-following model is a kind of responsestimulus model, in which a follower tries to maintain a space gap with its leader. Early car-following models were proposed by Reuschel and Pipes $[14,15]$. In order to account for the time lag, in 1958, Chandler et al. suggested an improved carfollowing model [16]:

$$
\ddot{x}_{n}(t+T)=\lambda\left[\dot{x}_{n+1}(t)-\dot{x}_{n}(t)\right],
$$

where $T$ is a response time lag and $\lambda$ is the sensitive coefficient. For a more realistic description, Newell presented the optimal velocity car-following model [17]:

$$
\ddot{x}_{n}(t+T)=V^{\text {opt }}\left(\Delta x_{n}(t)\right) \text {, }
$$

where the function $V^{\mathrm{opt}}$ is $V^{\mathrm{opt}}\left(\Delta x_{n}(t)\right)=v_{0}\left(1-\exp \left[-\left(\Delta x_{n}-\right.\right.\right.$ $\left.\left.\left.x_{c}\right) /\left(v_{0} T_{f}\right)\right]\right), \Delta x_{n}, x_{c}$, and $v_{0}$ are the headway distance, safe distance, and the desired velocity, respectively, and $T_{f}$ is a safe time interval characterizing the car-following behavior.

In 1995, Bando et al. proposed a famous OV car-following model which is written as follows [4]:

$$
\ddot{x}_{n}(t)=\frac{1}{\tau}\left(V^{\mathrm{opt}}\left(\Delta x_{n}(t)\right)-v_{n}(t)\right) .
$$

Here, $V^{\text {opt }}$ is the desired optimal velocity function. $\tau$ is the relaxation time. The desired optimal velocity of the $n$th vehicle $V_{n}^{\text {opt }}$ is derived as follows:

$$
V_{n}^{\text {opt }}=\frac{v_{\max }}{2}\left\{\tanh \left[\Delta x_{n}(t)-S_{m}\right]+\tanh \left(S_{m}\right)\right\} .
$$

Here, $v_{\max }$ is the maximum velocity of vehicles, and $\Delta x_{i}(t)$ is the distance between the $i$ th vehicle and the $(i+1)$ th vehicle. $S_{m}$ is called the minimum safety distance.

In fact, the OV car-following model belongs to the response-stimulation equation. The stimulation is the difference between the velocity of vehicle and optimal velocity. In the past decades, OV car-following model attracts more and more concerns because not only it is simple in numerical calculation, but also it can be analyzed by using iteration equation. For instance, the kink-antikink waves can be obtained.

\section{Our Proposed Model}

Usually, from road to railway and civil aviation, vehicles encounter many disturbances of uncertain factors, for example, the disturbance of weather, and the error of driver's judgment. To a larger extent, these uncertain factors directly affect vehicles' movement. In existing research reports, the influences of these factors are always ignored, especially in the railway traffic. This directly leads to the fact that the constructed simulation model or mathematical computations are not consistent with some realistic observations. Furthermore, some theoretical results using existing models or methods have unrealistic defects.

In our proposed model, the headway distance $\Delta x_{n}$ is considered as a fuzzy variable. In railway traffic, the headway distance $\Delta x_{n}$ is actually the following gap, and it can be obtained by calculating the distance between two successive trains. In general, the position of train must be detected and transmitted to control center uninterrupted. Because of the errors caused by the detection devices, environments, or communication system, the position of train cannot be obtained accurately. This makes the headway distance (i.e., the following gap) $\Delta x_{n}$ be an uncertain value. In this paper, $\Delta x_{n}$ is assumed to be a random value. It is divided into two parts: one part is actual value $\Delta x_{n a}$ and the other is error $x_{\mathrm{er}}$. 


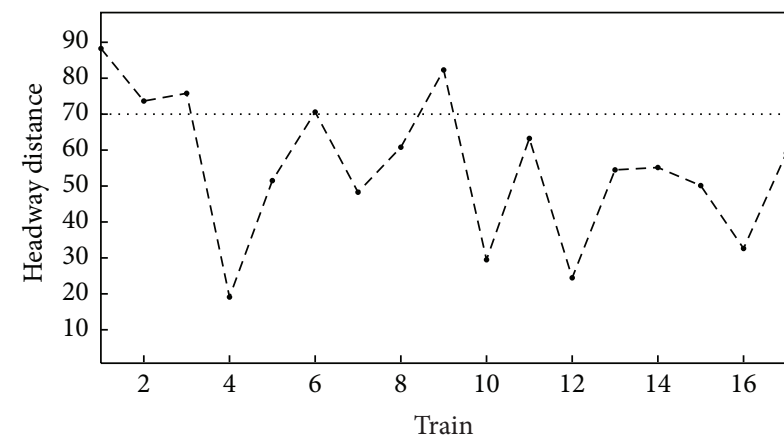

(a)

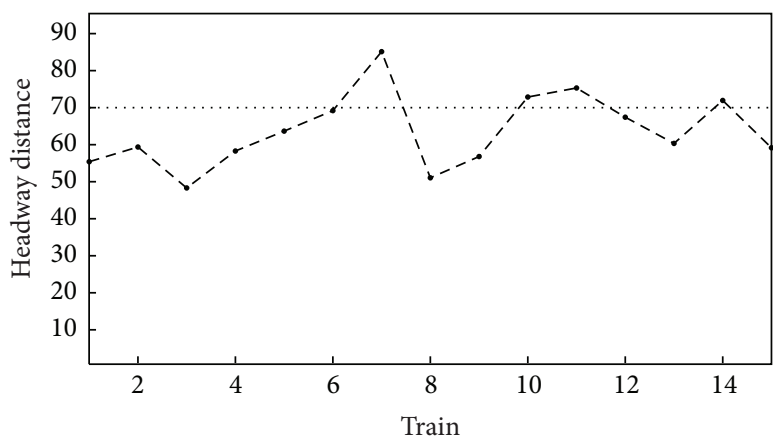

(b)

Figure 2: The distribution of headway distance for (a) $d_{m}=60$ and (b) $d_{m}=20$.

Because it is difficult to predict error value, we take $x_{\mathrm{er}}$ as a random value, and it is calculated by $x_{\mathrm{er}}=d_{m} n_{r}$; here, $n_{r}$ is a random number. So, $\Delta x_{n}$ can be taken as $\Delta x_{n}=\Delta x_{n a}+x_{\mathrm{er}}$. Here, $\Delta x_{n a}$ represents the accurate headway distance between the $n$th vehicle and the $(n+1)$ th vehicle. $x_{\text {er }}=d_{m} n_{r}, d_{m}$ represents the maximum error of headway distance, and $n_{r}$ is a random number in the region $\left[\begin{array}{ll}-1 & 1\end{array}\right]$.

In this paper, we apply expected value principle in fuzzy theory to deal with the fuzzy variable $\Delta x_{n}$. Firstly, let $x_{\mathrm{er}}$ change randomly for $N_{m}$ times from the formula of (5), so that $N_{m}$ values of $V^{\text {opt }}$ can be obtained. Averaging these $N_{m}$ values of $V^{\text {opt }}$, a mean value $V_{r a}$ is given. Repeating the above step for $N_{n m}$ times, and then averaging $N_{n m}$ values of $V_{r a}$, a desired optimal velocity $V_{r p}$ is obtained.

At time $t$, the executed process of the proposed model can be described as follows.

Step 1. Initial parameters are $N_{r m}=0$ and $V_{r m}=0$.

Step 2. Initial parameters are $N_{r}=0$ and $V_{r}=0$.

Step 3. Let $x_{\text {er }}$ change randomly: $N_{r}=N_{r}+1$.

Step 4. According to (5), calculating the optimal velocity $V^{\text {opt }}, V_{r}=V_{r}+V^{\text {opt }}$.

Step 5. If $N_{r}<N_{m}$, go to Step 3; else $V_{r a}=V_{r} / N_{m}, N_{r m}=$ $N_{r m}+1$, and $V_{r m}=V_{r m}+V_{r a}$.

Step 6. If $N_{r m}<N_{n m}$, go to Step 2; else $V_{r p}=V_{r m} / N_{n m}$.

In our method, the boundary condition is open. From the site $x=1$ to the site $x=S_{m}$, if there is no vehicle with the region $\left[0, S_{m}\right]$, a vehicle with the velocity $v_{n}=0$ is created at the site $x=1$. The newborn vehicle immediately moves according to the proposed model. At the site $L$, vehicles simply move out of the simulated system. $L$ is the length of the considered simulated system.

\section{Numerical Computation}

We use the proposed OV car-following model to simulate the evolution of traffic flow in single-lane railway traffic. Here, all vehicles must move and only the minimum safety distance $S_{m}$ is kept among them. In railway traffic, the minimum safe distance is calculated by $S_{m}=\tau V_{\max }+V_{\max }{ }^{2} / 2 b_{\max }+\Delta x_{\operatorname{mag}}$. Here, $\tau$ is relaxation time, $V_{\max }$ is maximum speed, $b_{\max }$ is the maximum deceleration, and $\Delta x_{\text {mag }}$ is margin which is fixed. According to this formula, when $V_{\max }$ and $b_{\max }$ are constant, $S_{m}$ is constant too. The length of the simulated system is set to be $L=2000$, and the maximum iteration time step is $T=$ 1000. A station is designed at the middle site of the simulated system. At the station, all vehicles should stop for the same time $T_{d}$ and then leave. $T_{d}$ is called the dwell time (red light time). The parameters $v_{\max }, S_{m}, \tau$, and $T_{d}$ are taken as 10, 70, 2.5 , and 5 , respectively.

In reality traffic, the safety factor is very important in which collisions between two vehicles must be avoided. This means that the distance between two successive vehicles must be larger than the minimum safety distance. In order to investigate the evolution of traffic flow under uncertain environment, we use the proposed OV car-following model to simulate the vehicle's movements. The uncertain environment is completed by $\Delta x_{n}=\Delta x_{n a}+x_{\mathrm{er}}$. After sufficient transient, we begin to record the traffic series of the headway distance at a given time $t,\left\{D_{i}\right\}$, where $D_{i}$ is the distance from the $i$ th vehicle to the $(i+1)$ th vehicle. Figure 2 shows the distribution of the headway distance $D_{i}$ at $t=600$. In Figure 2, the minimum safety distance $S_{m}$ is indicated by the dotted line, and the simulation results are drawn by the dashdot line. Figure 2(a) displays the fact that the distribution of headway distance is less than $S_{m}$, and most of headway distances are within the region $[20,60]$. When $d_{m}$ is equal to 20, the distribution of headway distance shown in Figure 2(b) is larger than 50. Comparing Figure 2(a) with Figure 2(b), we can conclude that as $d_{m}$ is larger, the probability of collisions among trains is higher. In this case, the safety of train becomes worse.

Applying excepted value principle, the train's velocity can be optimized. So we use such a method to deal with the vehicle's velocity under uncertain condition. The simulation environment is the same as that adopted in Figure 2(a). Figure 3 shows the distributions of optimized headway distance under $d_{m}=60$. In Figure 3, we can see that although most of the simulation results are still smaller than $S_{m}$, the 


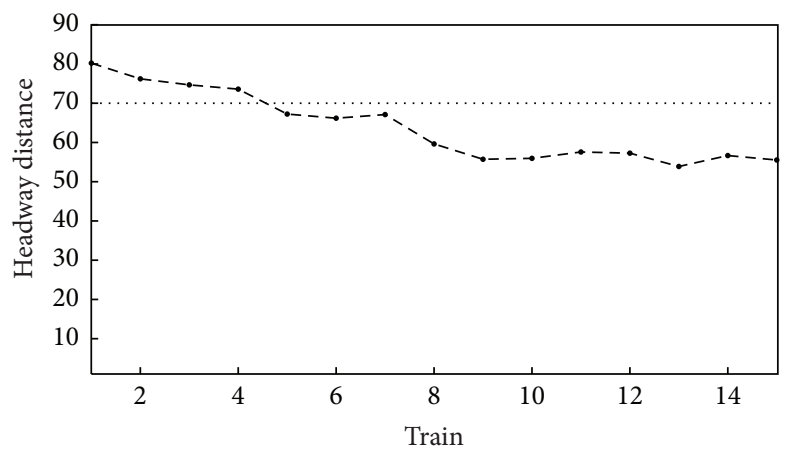

FIgURE 3: The distribution of headway distance under the excepted value principle for $d_{m}=60$.

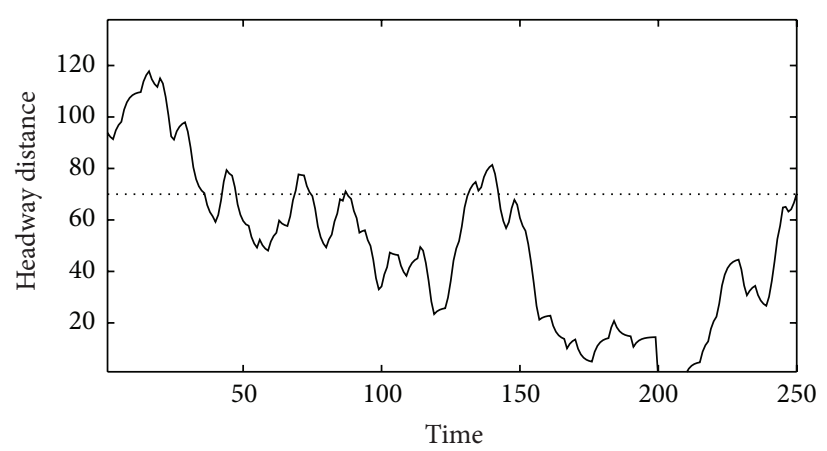

(a)

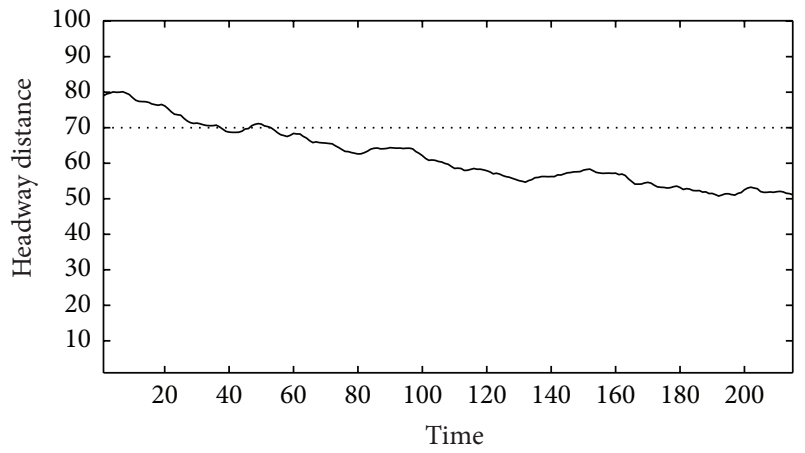

(b)

FIgURE 4: The distribution of headway distance of a single tracked train for $d_{m}=60$ (a) without the excepted value principle and (b) with the excepted value principle.

values of headway distance vary slowly within the region $[50,80]$. Compared to Figure 2(a), it is obvious that our proposed model which uses the expected value principle can effectively improve the safety of train.

In order to further study the safety of train, the headway distance $D(t)$ of one tracked train is measured. Here, $D(t)$ is the distance from the tracked train to its leading train at time $t$. Figure 4 shows the simulation results of the tracked train which departs at the time $t=600$. From Figure 4(a), it is clear that most of distance $D(t)$ is smaller than the minimum safety distance. Moreover, within some time intervals, the headway distance of tracked vehicle is zero. In this case, collision occurs. The result demonstrates that train travelling is very dangerous under the uncertain condition. Figure 4(b) shows the headway distance $D(t)$ of the tracked train. Here, although $D(t)$ is still smaller than the minimum safety distance, they are larger than 50. Moreover, it also shows that the values of $D(t)$ change slowly. In this case, the travelling comfort is also improved.

In the theory of traffic flow, the stability of traffic model is a major character. In general, the stability of traffic model is described by the variation amplitude of the headway distance which is got by calculating the difference between the maximum value and average value of the headway distance. Figure 5(a) shows the variation amplitude of a tracked train at all time under the uncertain environment. Here the variation amplitude of the headway distance is within the region $[0,50]$. However, from Figure 5(b), the amplitude of the headway distance varies within the region $[0,20]$, and most of the values are less than 10 . Numerical results indicate that not only the collision can be avoided, but also the fluctuation of amplitude is small compared with Figure 5(a). This means that the stability of the tracked train simulated using our proposed car-following model is improved.

In order to further study the stability character of train travelling, the variation amplitude of headway distance of all trains at a given time is measured. Figure 6 shows the results in which the variation amplitude of headway distance is obtained at $t=600$. The variation amplitude of the headway distance using the excepted value principle shown in Figure 6(b) is small and slow, and it is less than that shown in Figure 6(a). In terms of the simulation results which are shown in Figure 6, we can conclude that train travelling is not stable under the uncertain factor, but the situation can be improved using our proposed model where the expected value principle is used.

We study the stability of one tracked train. The variation acceleration of the tracked train which departs at $t=600$ is measured. Figure 7(a) shows that the acceleration of the tracked train varies sharply and frequently. It demonstrates that the tracked train cannot obtain stable travelling. From Figure $7(\mathrm{~b})$, the amplitude of the acceleration is within a small 


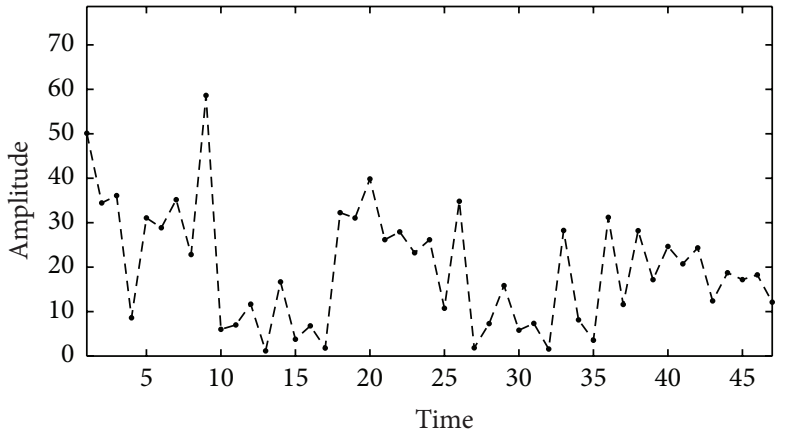

(a)

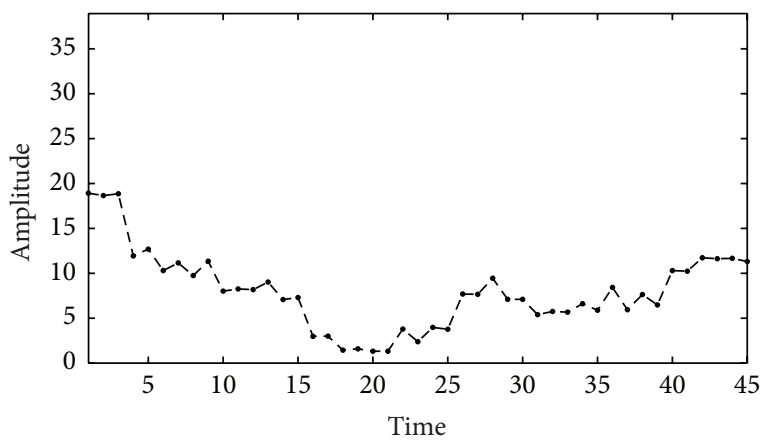

(b)

FIGURE 5: The variation amplitude of headway distance of a tracked train for $d_{m}=60$ (a) without the excepted value principle and (b) with the excepted value principle.

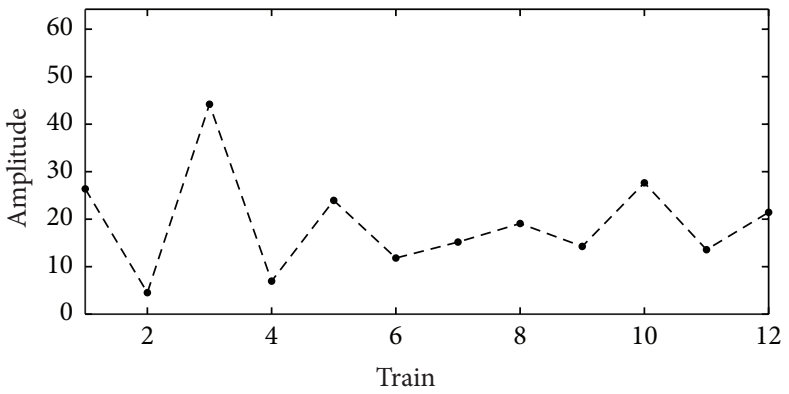

(a)

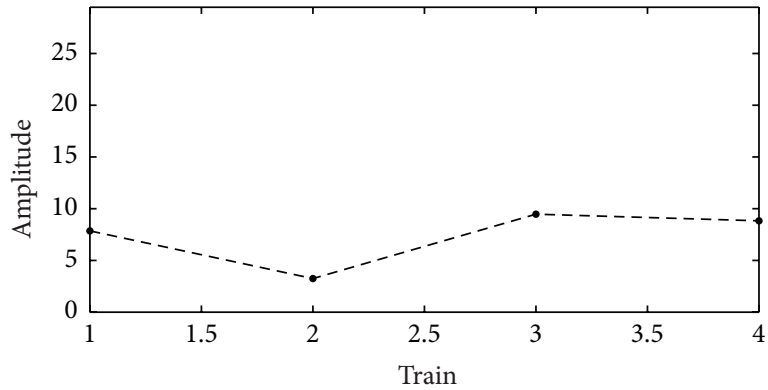

(b)

FIGURE 6: The variation amplitude of headway distance of all trains for $d_{m}=60$ (a) without the excepted value principle and (b) under the excepted value principle.

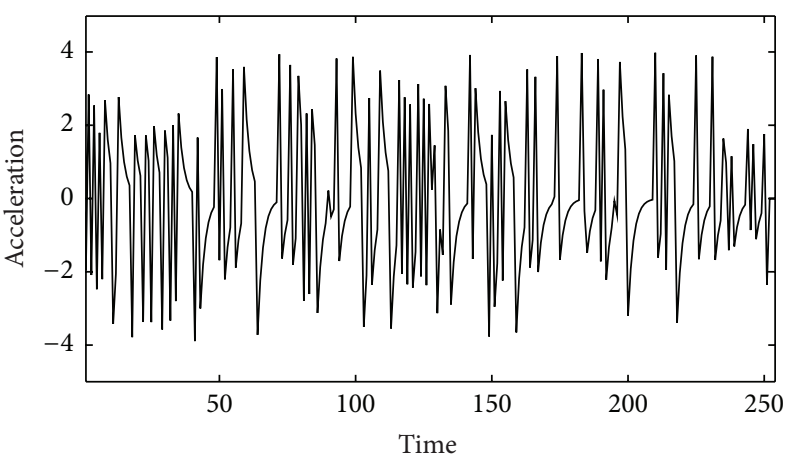

(a)

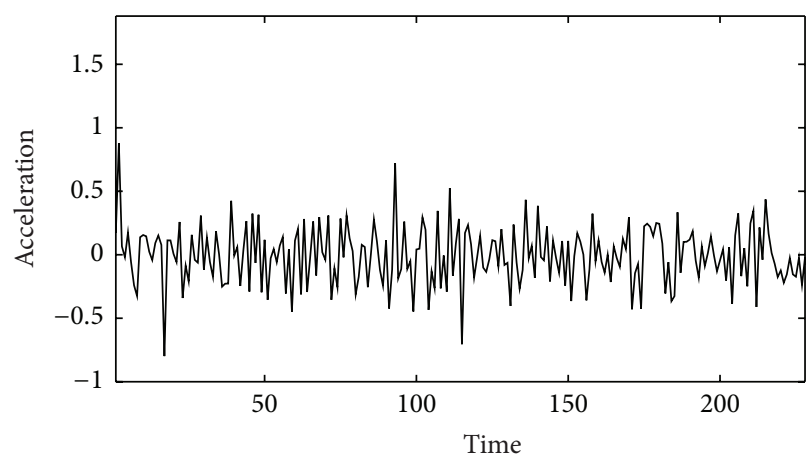

(b)

Figure 7: How the acceleration varies with the time for $d_{m}=60$ (a) without the excepted value principle and (b) with the excepted value principle.

region $[-1,1]$. Most of the measured values are far smaller than 0.5 . This means that the driver only needs to adjust the velocity of the tracked train simulated using our proposed model, and the stability of train travelling can be enhanced.

Relaxation time $\tau$ is one of the major parameters of car-following model which describes the response extent of the driver. In general, the driver's response greatly affects the train travelling. In theory, as the relaxation time is larger, the response of driver is slower. Figure 8 shows the variation amplitude of headway distance of one tracked train for different relaxation time. Here, the headway distance is uncertain. Comparing Figure 8(a) with Figure 8(b), it is clear that the values of amplitude shown in Figure 8(a) are larger than that shown in Figure 8(b). It indicates that the stability of train traveling at $\tau=1$ is better than $\tau=4$. The simulation results are consistent with some observation results.

When the excepted value principle is applied to our proposed car-following model, how the variation amplitude changes with the relaxation time varies. Figure 9 shows the variation amplitude of one single tracked train for different 


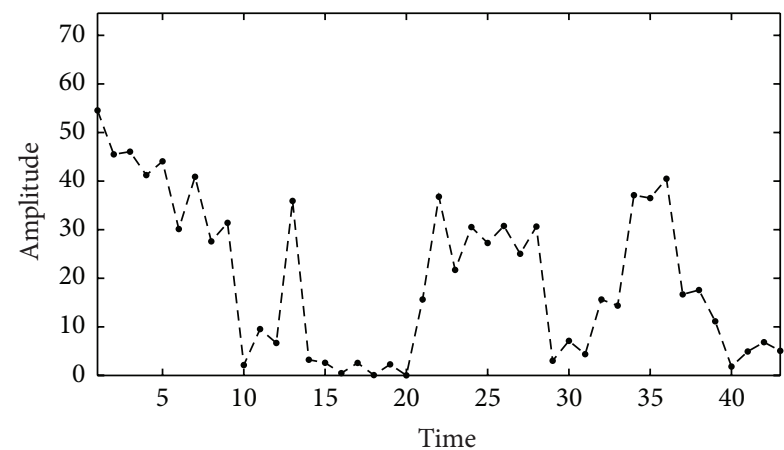

(a)

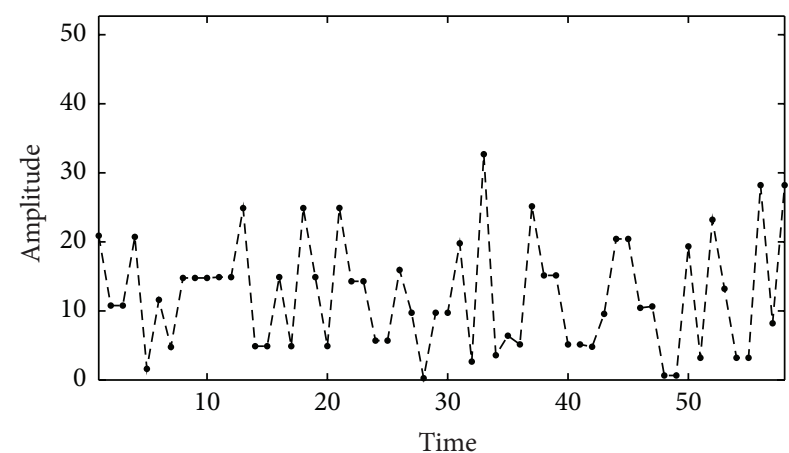

(b)

FIGURE 8: The variation amplitude of headway distance of one tracked train for $d_{m}=60$. (a) $\tau=4$ and (b) $\tau=1$.

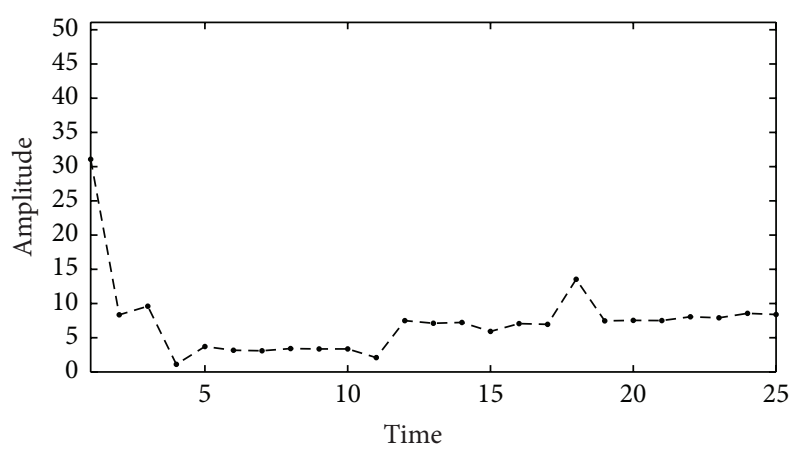

(a)

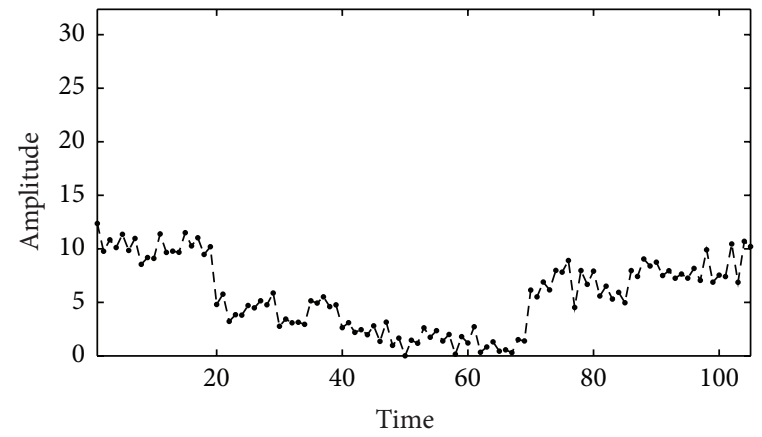

(b)

FIGURE 9: The variation amplitude of headway distance under the excepted value principle for (a) $\tau=4$ and (b) $\tau=1$.

time $\tau$, where the excepted value principle is used. Here we can see that there is no obvious oscillation which is similar to that shown in Figure 8. Moreover, we observe that the overall values of Figure 9 (b) are smaller than that shown in Figure 9(a). The results further demonstrate that the quick response of driver contributes to the stability of train travelling.

In Figures 8 and 9, we find that even if one train has a greater acceleration, the stability of system consisting of many trains is good. The reason is that, in our paper, the stability of train movement is described by the difference among the headway distances of tracked trains. So the stability is used to investigate the systematic character of many trains.

\section{Conclusion}

In summary, we use an extended OV car-following model to simulate the evolution of traffic flow under uncertain condition. Here, the headway distance of train is regarded as fuzzy variable. The simulation results demonstrate that not only the disturbance of uncertain factors can be overcome effectively, but also the stability character of our proposed model can be improved.

We also discuss that the relaxation time affects the stability based on the proposed model. The stability is improved with the shortening of the relaxation time. Because optimizing of the excepted value principle weakens the influence of error headway distance, the improvement of stability under the excepted principle is not very obvious.

Here, we only take a simple uncertain factor into account; however, some complex factors, such as the resistance force of vehicle movement and the disturbance of wind and sand, are not considered. Even so, we provide a good way to handle the uncertain factor in the theory of traffic flow. We will do them in our future works. Moreover, our results are useful for optimizing the design and plan of urban traffic system.

At present, there are some better extensions of the $\mathrm{OV}$ model, such as the FVD [18] model and MMVD model [19]. Although they are mainly applied for the road traffic, they may be good for solving some railway traffic problems.

\section{Conflict of Interests}

The authors declare that there is no conflict of interests regarding the publication of this paper.

\section{Acknowledgment}

This work was supported by the Fundamental Research Funds for the Central Universities (no. 2014JBM109). 


\section{References}

[1] T. Komatasu and S. Sasa, "Kink solution charactering traffic congestion," Physical Review E, vol. 52, pp. 5574-5581, 1995.

[2] M. Muramatsu and T. Nagatani, "Soliton and kink jams in traffic flow with open boundaries," Physical Review E, vol. 60, no. 1, pp. 180-187, 1999.

[3] H. X. Ge, S. Q. Dai, and L. Y. Dong, "An extended car-following model based on intelligent transportation system application," Physica A: Statistical Mechanics and Its Applications, vol. 365, no. 2, pp. 543-548, 2006.

[4] M. Bando, K. Hasebe, K. Nakanishi, A. Nakayama, A. Shibata, and Y. Sugiyama, "Phenomenological study of dynamical model of traffic flow," Journal de Physique I, vol. 5, no. 11, pp. 1389-1399, 1995.

[5] D. Helbing and B. Tilch, "General force model of traffic dynamics," Physical Review E, vol. 58, no. 1, pp. 133-138, 1998.

[6] R. Jiang, Q. S. Wu, and Z. J. Zhu, "Full velocity difference model for a car-following theory," Physical Review E, vol. 64, no. 1, Article ID 017101, 2001.

[7] S. K. Li, L. X. Yang, Z. Y. Gao, and K. P. Li, "Stabilization strategies of a general nonlinear car-following model with varying reaction-time delay of the drivers," ISA Transactions, vol. 53, no. 6, pp. 1739-1745, 2014.

[8] S. F. Zheng, J. D. Cao, X. M. Lian, and K. P. Li, "Urban pickup and delivery problem considering time-dependent fuzzy velocity," Computers \& Industrial Engineering, vol. 60, no. 4, pp. 821-829, 2011.

[9] A. Chen and Z. Zhou, "The $\alpha$-reliable mean-excess traffic equilibrium model with stochastic travel times," Transportation Research Part B: Methodological, vol. 44, no. 4, pp. 493-513, 2010.

[10] F. Zheng and H. Van Zuylen, "Uncertainty and predictability of urban link travel time: delay distribution-based analysis," Transportation Research Record, vol. 2192, pp. 136-146, 2010.

[11] L. X. Yang, K. P. Li, and Z. Y. Gao, "Train timetable problem on a single-line railway with fuzzy passenger demand," IEEE Transactions on Fuzzy Systems, vol. 17, no. 3, pp. 617-629, 2009.

[12] M. Y. Wei, X. Li, and L. Yu, "Time-dependent fuzzy random location-scheduling programming for hazardous materials transportation," Transportation Research Part C: Emerging Technologies, vol. 57, pp. 146-165, 2015.

[13] L. X. Yang, X. S. Zhou, and Z. Y. Gao, "Credibility-based rescheduling model in a double-track railway network: a fuzzy reliable optimization approach," Omega, vol. 48, pp. 75-93, 2014.

[14] A. Reuschel, "Fahrzengbewegungenin der kolonne oesterr," in Der Österreichische Ingenieur- und Architekten-Verein, vol. 4, pp. 193-215, 1950.

[15] L. A. Pipes, "An operational analysis of traffic dynamics," Journal of Applied Physics, vol. 24, no. 3, pp. 274-281, 1953.

[16] R. E. Chandler, R. Herman, and E. W. Montroll, "Traffic dynamics: studies in car following," Operations Research, vol. 6, no. 2, pp. 165-184, 1958.

[17] G. F. Newell, "Nonlinear effects in the dynamics of car following," Operational Research, vol. 9, no. 2, pp. 209-229, 1961.

[18] R. Jiang, Q. S. Wu, and Z. J. Zhu, "Full velocity difference model for a car-following theory," Physical Review E, vol. 64, no. 1, Article ID 017101, 4 pages, 2001.

[19] T. Wang, Z. Y. Gao, and X. M. Zhao, "Multiple velocity difference model and its stability analysis," Acta Physica Sinica, vol. 55, no. 2, pp. 634-640, 2006. 


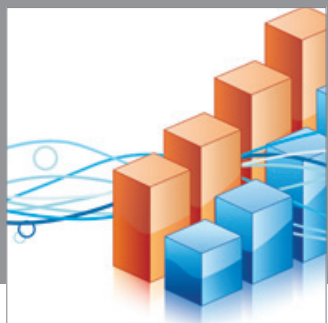

Advances in

Operations Research

mansans

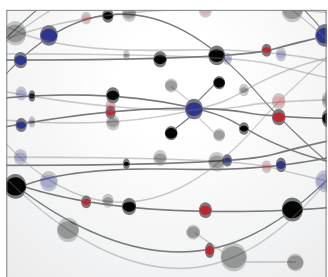

The Scientific World Journal
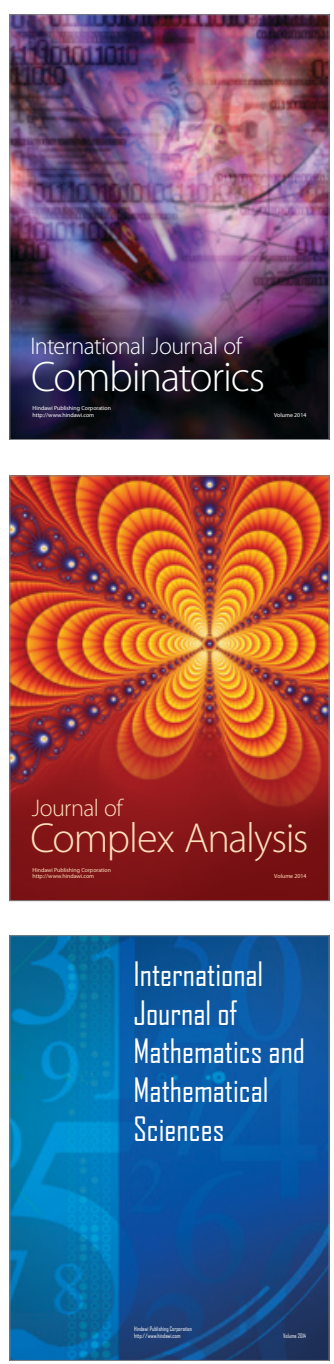
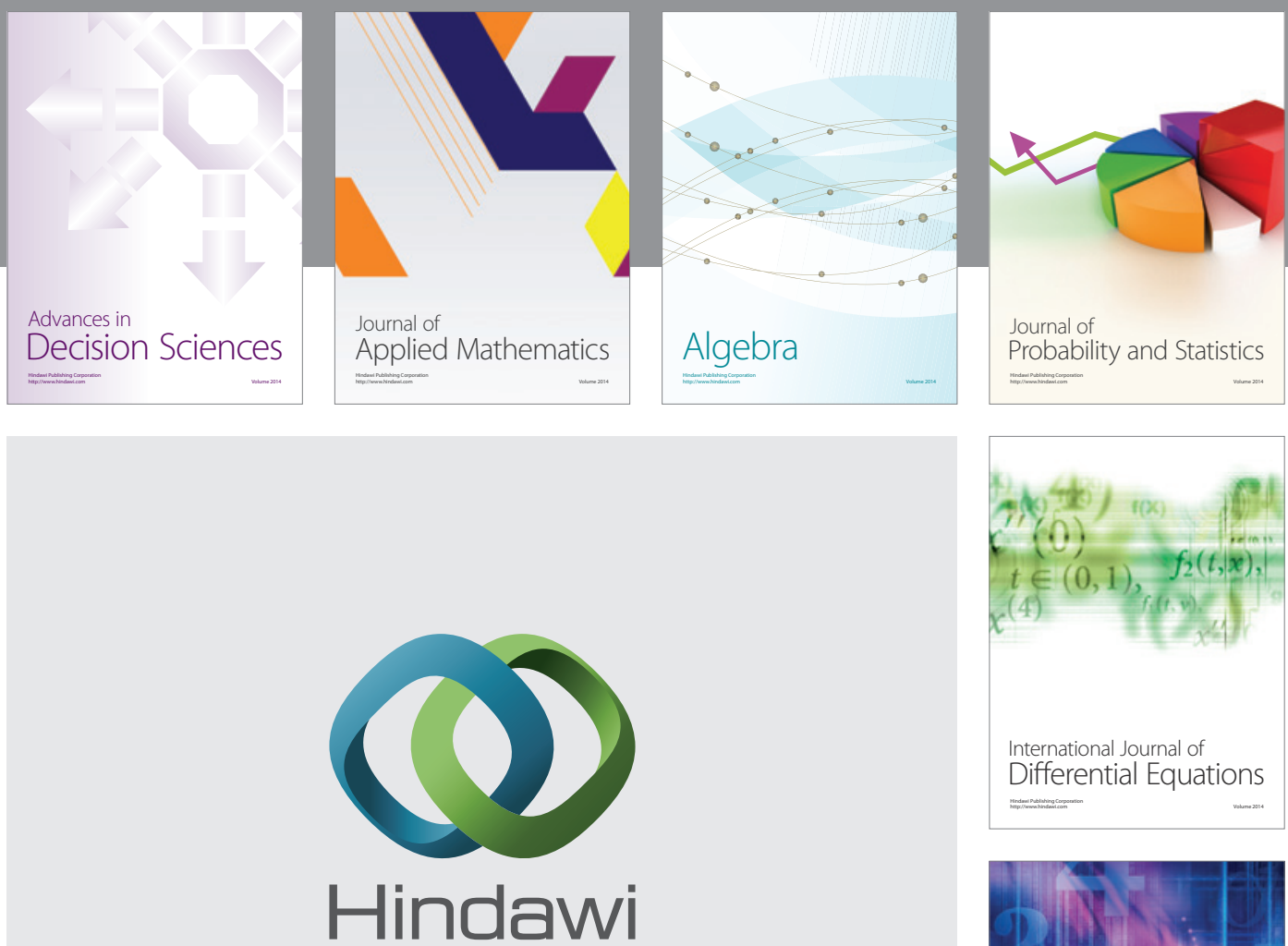

Submit your manuscripts at http://www.hindawi.com
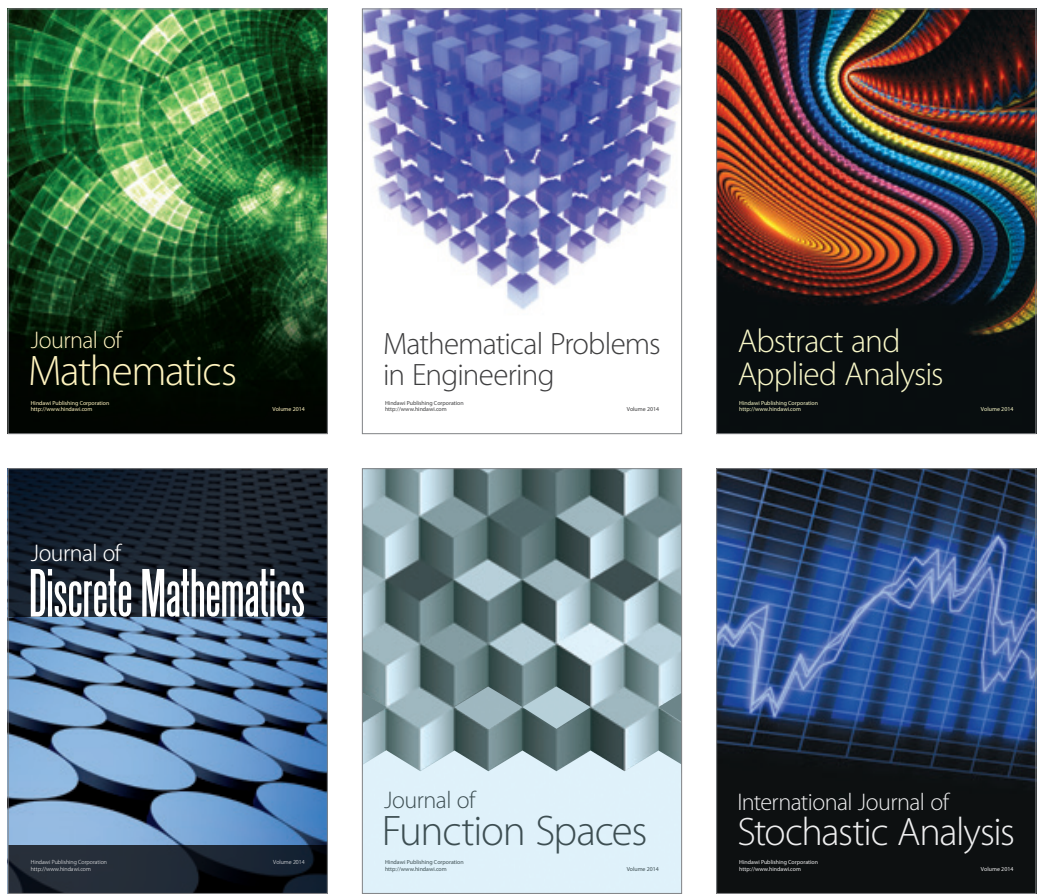

Journal of

Function Spaces

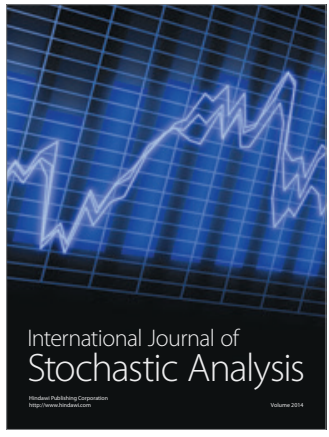

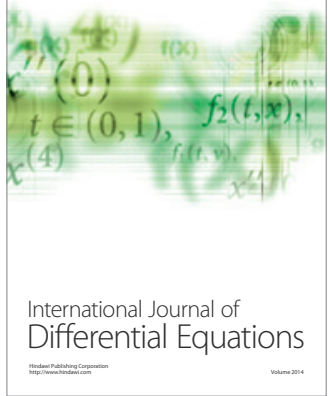
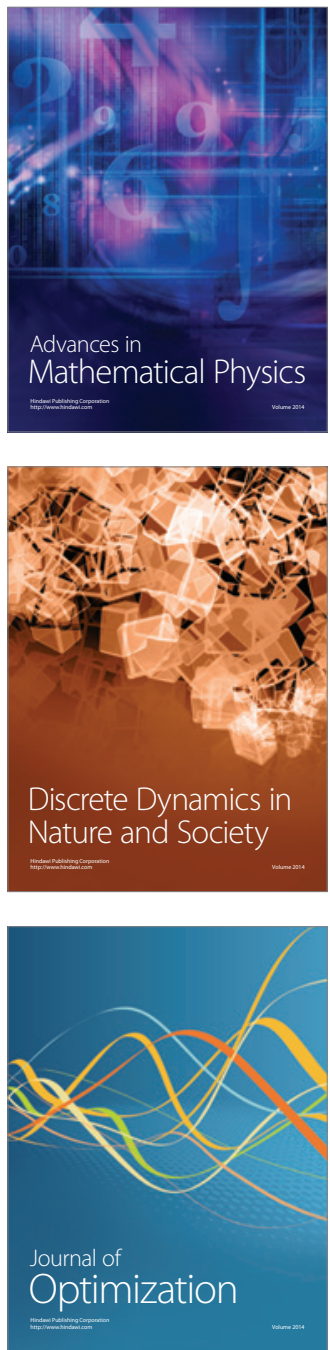